\title{
Enhancement of oxidation rate of a-Si nanoparticles during dehydrogenation
}

\author{
D. Das, J. Farjas, and P. Roura ${ }^{\text {a) }}$ \\ GRM, Departament de Física, Universitat de Girona, Campus Montilivi, E17071 Girona, Catalonia, Spain
}

G. Viera and E. Bertran

FEMAN, Departament de Física Aplicada i Optica, Universitat de Barcelona, Av. Diagonal 647, E08028 Barcelona, Catalonia, Spain

(Received 29 May 2001; accepted for publication 12 September 2001)

\begin{abstract}
Oxidation of amorphous silicon $(a-\mathrm{Si})$ nanoparticles grown by plasma-enhanced chemical vapor deposition were investigated. Their hydrogen content has a great influence on the oxidation rate at low temperature. When the mass gain is recorded during a heating ramp in dry air, an oxidation process at low temperature is identified with an onset around $250{ }^{\circ} \mathrm{C}$. This temperature onset is similar to that of hydrogen desorption. It is shown that the oxygen uptake during this process almost equals the number of hydrogen atoms present in the nanoparticles. To explain this correlation, we propose that oxidation at low temperature is triggered by the process of hydrogen desorption. (C) 2001 American Institute of Physics. [DOI: 10.1063/1.1420533]
\end{abstract}

The microstructure and properties of nanosized amorphous silicon $(a-\mathrm{Si})$ powders have been extensively studied during last few years ${ }^{1-5}$ for their possible application in microelectronic devices. The growth of nanostructured $a-\mathrm{Si}$ thin films (also called polymorphous $\mathrm{Si}$ ) has also been of considerable interest because of its possible application in multijunction $a$-Si solar cells by virtue of its enhanced properties for photovoltaic conversion. ${ }^{6}$ They are currently grown by chemical vapor deposition (CVD) techniques in which the process parameters are chosen in such a way that the small particles (nanometric size) that grow in the plasma are deposited onto the substrate and are covered by $a$-Si network. Consequently, the final structure consists of amorphous or crystalline nanoparticles embedded in an amorphous matrix. ${ }^{7}$ One of the major drawbacks of this material could be its oxidation tendency Thus, the oxidation mechanism at low temperature should be investigated.

In this letter, we report the results of our experiments done with the aim of understanding the role of hydrogen in the oxidation mechanisms of $a$-Si nanoparticles grown by plasma-enhanced chemical vapor deposition (PECVD). In the case of glow-discharge $a$-Si films, a significant oxygen uptake at room temperature after hydrogen desorption experiments was observed. ${ }^{8}$ This effect was more important with a greater initial hydrogen content presumably due to the higher density of dangling bonds that survived after cooling down to room temperature. In our experiments, oxidation is monitored during dehydrogenation. ${ }^{9}$ Preliminary results ${ }^{10}$ indicated that oxidation could be enhanced because, in these conditions, the dangling bond density would be higher. This hypothesis has been analyzed now with greater detail.

Silicon nanoparticles were grown at room temperature by PECVD from square wave-modulated $\mathrm{rf}$ discharges of silane. ${ }^{3}$ Transmission electron microscopy observations ${ }^{11}$ have revealed that the particles are mostly spherical with mean $20 \mathrm{~nm}$ diameter whereas electron diffraction patterns

\footnotetext{
${ }^{a}$ Author to whom all correspondence should be addressed; electronic mail: pere.roura@udg.es
}

show that they are amorphous. Another important structural characteristic is their high hydrogen content that comes from the silane precursor gas.

Hydrogen and oxygen content has been determined by elementary analysis (EA). The EA measurements were corrected for any contribution from extraneous sources by subtracting the blank signal obtained without sample. In fact, with EA, one directly obtains the hydrogen content with an accuracy of 0.02 hydrogen atoms per $\mathrm{Si}$ atom. To establish the $\mathrm{Si}$ and $\mathrm{O}$ content, the EA technique was complemented with the mass gain of the powder after complete oxidation. In this way, we could know the chemical composition of the powders before oxidation experiments, which can be written as $\mathrm{O}_{0.22} \mathrm{Si}_{1} \mathrm{H}_{y}(0.01<y<0.45)$. The range of hydrogen content values was obtained after heat treatment in an inert atmosphere of the as-grown powder. This significant oxygen content is due to the instantaneous oxidation occurring when the CVD chamber is first opened to an atmosphere. Despite the subsequent exposure of the sample to air at room temperature, its oxygen content did not increase significantly.

Dehydrogenation and oxidation experiments have been performed by differential scanning calorimetry (DSC) and thermogravimetry (TGA). High purity oxygen or synthetic air were used as the oxidation gas. When necessary, the oxygen partial pressure was reduced by dilution with nitrogen. Dehydrogenation experiments were performed in an inert atmosphere $\left(\mathrm{N}_{2}\right.$ or $\left.\mathrm{Ar}\right)$. DSC and TGA thermograms were routinely corrected by the apparatus baseline. Additionally, in order to assess the reliability of mass gain curves, the total mass change recorded by TGA was compared with the difference of masses measured at room temperature before and after every oxidation experiment.

Figure 1 shows the rate of mass gain of the nanoparticles due to oxidation in synthetic air during heating with a steady ramp of $20^{\circ} \mathrm{C} / \mathrm{min}$ for different hydrogen contents ( $y$ values). A low temperature oxidation process is identified as having an onset at about $230{ }^{\circ} \mathrm{C}$ and a maximum oxidation rate around $350^{\circ} \mathrm{C}$. The contribution of this process diminishes when hydrogen content is lowered and becomes almost neg- 


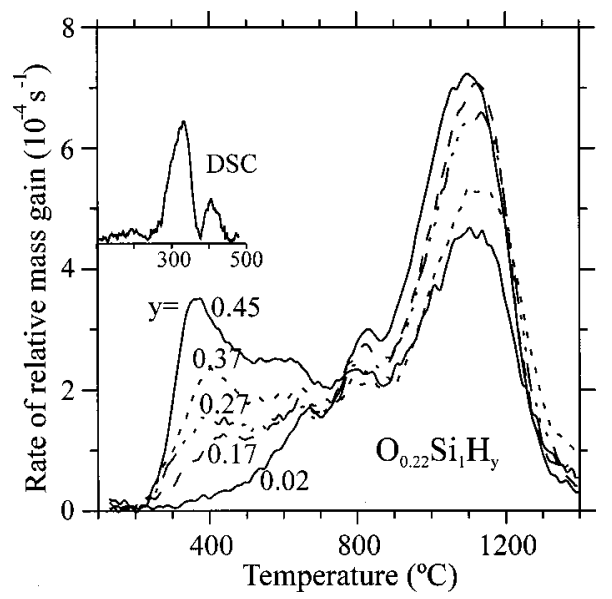

FIG. 1. Rate of oxidation of $a$-Si nanoparticles during heating with a steady ramp for different initial hydrogen contents. The strength of the lowtemperature peak gradually decreases with the lowering of hydrogen content of the powder. Inset: heat evolved during a heating ramp in inert atmosphere. The peaks correspond to dehydrogenation processes.

ligible at $y=0.02$. At higher temperature, the usual oxidation process leading to the formation of a protective layer of silica takes place which leads to complete oxidation. This process follows the standard theory of Si oxidation. ${ }^{12}$ This fact can be deduced from the constant mass gain (the area below the curves) reached at $1400{ }^{\circ} \mathrm{C}$ for any curve and has been confirmed by IR spectroscopy.

In view of the relationship between hydrogen content and oxidation at low temperature, the dehydrogenation process has been studied with some detail. The heat evolved during annealing of the as-grown powder with a steady heating ramp in argon atmosphere is shown in the inset of Fig. 1. Two exothermic peaks are resolved which are known to correspond to dehydrogenation. ${ }^{13}$

In a recent article ${ }^{14}$ we have reported a low-temperature oxidation process that takes place in hydrogenated $\mathrm{SiC}$ nanoparticles grown by PECVD. It was demonstrated that this oxidation was similar to the oxidation of polymers in the sense that it proceeded throughout the entire volume of a nanoparticle, homogeneously, without the formation of any protective layer at the surface. In contrast to the oxidation of metals and crystalline semiconductors, ${ }^{12}$ there, oxidation was not diffusion controlled. In preliminary experiments done in Si nanoparticles, ${ }^{10}$ the mass gain transients show an initial nonzero slope at $320^{\circ} \mathrm{C}$. Hence, in Si nanoparticles, oxidation at low temperature is not diffusion controlled, too. However, in contrast to the case of $\mathrm{SiC}$, in $\mathrm{Si}$ nanoparticles hydrogen presumably plays a more important role because oxidation takes place during dehydrogenation, whereas in $\mathrm{SiC}$, oxidation occurs at a lower temperature.

The onset and peak temperatures are very similar for oxidation and dehydrogenation processes (Fig. 1). It seems as if oxidation was triggered by dehydrogenation. Thus, we propose the following two-step mechanism: (step A) is based on the widely accepted fact that dehydrogenation of two neighboring $\mathrm{Si}-\mathrm{H}$ bonds takes place at a low temperature where the simultaneous formation of a $\mathrm{H}_{2}$ molecule considerably reduces the activation energy of the process. ${ }^{16}$ Then, the two dangling bonds left behind offer a highly reactive Downloaded 26 Apr 2010 to 84.88.138.106. Redistribution subject

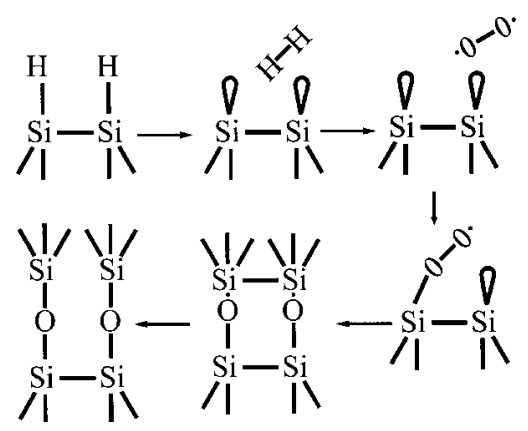

FIG. 2. Mechanism leading to oxygen incorporation after dehydrogenation. Oxygen can diffuse into the particles and react with one dangling bond. Due to the intrinsic instability of the peroxide group, two $\mathrm{Si}-\mathrm{O} \bullet$ groups will result and two unpaired electrons. These electrons induce $\mathrm{Si}-\mathrm{Si}$ bond break down and an interstitial oxygen $(\mathrm{Si}-\mathrm{O}-\mathrm{Si})$ formation.

site for oxygen atoms ready incorporation before substantial dangling bond recombination (step B).

Although it is difficult to know the details of oxygen incorporation from our results, we tentatively propose step B as shown in Fig. 2. A high hydrogen content in $a$-Si results in a structure of interconnected internal voids ${ }^{15}$ which may allow gases diffusion. Consequently, we consider that molecular oxygen can diffuse into the particles and react with one dangling bond. [Any initial reaction with both dangling bonds with formation of a peroxide bridge $(\mathrm{O}-\mathrm{O})$ between both $\mathrm{Si}$ atoms is prevented because it requires a great bending of $\mathrm{Si}-\mathrm{O}$ bonds.] Then, due to the intrinsic instability of the peroxide group, two $\mathrm{Si}-\mathrm{O} \cdot$ groups will result and the two unpaired electrons can be naturally allocated into the empty $d$ orbitals of saturated Si-atoms. Finally, these additional electrons can induce a $\mathrm{Si}-\mathrm{Si}$ bond to break down so that an interstitial oxygen $(\mathrm{Si}-\mathrm{O}-\mathrm{Si})$ is formed. Hence, one hydrogen atom is replaced by one oxygen atom.

Additional experimental support to this oxidation mechanism is given in the following.

(a) Onset temperature: The oxidation onset temperature has the same value irrespective of $\mathrm{H}$ amount (Fig. 1). This is as expected, due to the fact that dehydrogenation is a first-order reaction process. ${ }^{13,16}$ So, oxidation will be always triggered at the same temperature.

(b) Oxygen uptake: The amount of oxygen incorporated almost equals the $\mathrm{H}$ content (Fig. 3). The data points of Fig. 3 have been obtained as follows. The oxygen incorporation during the low-temperature oxidation process can be calculated from the area under the first oxidation peak in Fig. 1 by considering lowest $\mathrm{H}$-content curve as the baseline. On the other hand, $\mathrm{H}$ content is known with accuracy from the EA. This nice correlation indicates that, in accordance with the proposed mechanism, when hydrogen evolves, one oxygen atom is incorporated for every $\mathrm{H}$ atom left.

(c) Dependence on oxygen partial pressure: In Fig. 3, two points have been included which correspond to oxidation experiments carried out with pure oxygen and oxygen partial pressure $\left(P_{\mathrm{O} 2}\right)$ of 0.028 . The great variation of $P_{\mathrm{O} 2}$ has a minor effect on the oxygen uptake. This means that, at these conditions (temperature and partial pressure) relaxation processes leading to dangling bond
license or copyright; see http://apl.aip.org/apl/copyright.jsp 


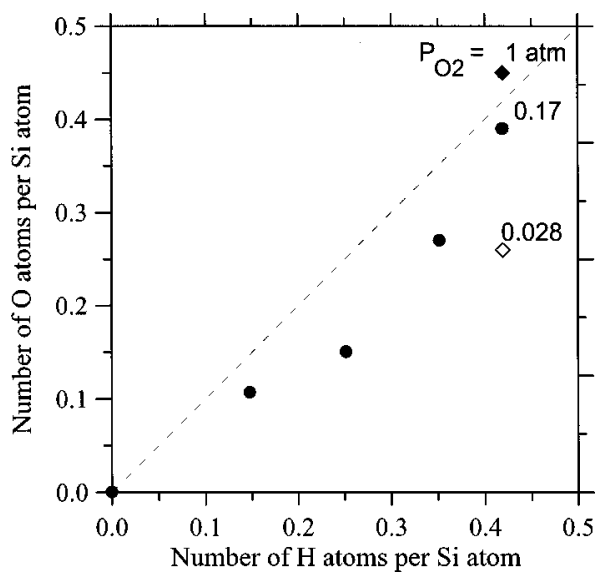

FIG. 3. Oxygen uptake as a function of initial hydrogen content at different oxygen partial pressures, $P_{\mathrm{O}_{2}}$. Oxygen incorporation has been obtained by integrating the low-temperature peak in Fig. 1. Hydrogen content has been directly achieved by applying EA. Oxygen uptake exhibits a very weak dependence on oxygen partial pressure.

recombination do not compete efficiently with oxidation. In other words, step B is much faster than recombination of dangling bonds.

(d) Oxide quality: If oxygen is incorporated throughout the whole volume without formation of a protective layer, then a suboxide of silicon will be formed and the atomic neighborhood of oxygen will be different from that in silica. This point can be assessed by looking at vibrational spectra obtained by IR spectroscopy (Fig. 4). The peak corresponding to the $\mathrm{Si}-\mathrm{O}-\mathrm{Si}$ vibrational mode centered at around $1100 \mathrm{~cm}^{-1}$ is compared for two samples oxidized during a heating ramp up to the same relative mass gain $(\Delta m / m \approx 0.09)$ before [curve Fig. 4(b)] and after [curve Fig. 4(c)] dehydrogenation. The overall IR absorption peak of the dehydrogenated and then oxidized sample [curve Fig. 4(c)] is similar to

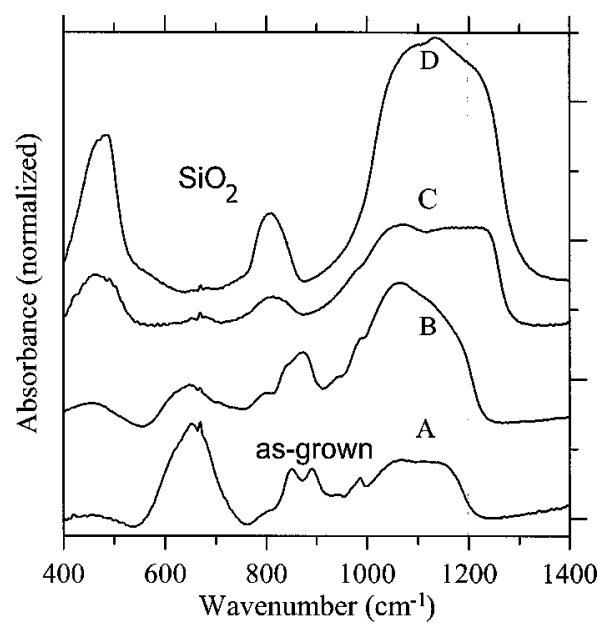

FIG. 4. Infrared absorbance spectra normalized to a initial mass of $0.1 \mathrm{mg}$. Spectra B and C correspond to hydrogenated and dehydrogenated powders, respectively, which have been oxidized up to the same mass gain. Note that spectrum $\mathrm{C}$ is more similar to that of silica nanoparticles. The vertical dashed line is drawn to make comparison easier. that of a completely oxidized sample [Fig. 4(d)], which corresponds to nanoparticles of pure silica. This result is as expected because, after dehydrogenation, oxidation proceeds through the formation of a silica layer. On the contrary, in oxidation without predehydrogenation [curve Fig. 4(b)] the IR peak lacks a high energy component $\left(1220 \mathrm{~cm}^{-1}\right)$ which corresponds to the $\mathrm{TO}_{4}$ mode in silica. ${ }^{17}$ In fact, the similarity of the peak at $1100 \mathrm{~cm}^{-1}$ between hydrogenated $\mathrm{Si}$ and $\mathrm{SiC}$ (Ref. 14) nanoparticles indicates that oxygen is incorporated as bridging oxygen ${ }^{18}$ and not as in silica. A suboxide has been formed.

In summary, the low-temperature oxidation of $a$-Si nanoparticles has been analyzed and we conclude that oxidation is triggered by hydrogen desorption. Oxygen is incorporated due to the dangling bonds that hydrogen leaves behind. At this point, we want to stress the similarity of this mechanism to the usual flame combustion of organic materials where it is well established that thermal decomposition affords free radicals which react with oxygen. The main difference is that, in the case of Si nanoparticles, the radicals are not really free but remain into the solid as Si-dangling bonds. Although this oxidation mechanism has been proved in $a$-Si nanoparticles, it can be extrapolated to CVD $a$-Si thin films because the hydrogen desorption process has the same kinetic parameters in both cases. ${ }^{9,13}$

This work has been supported by the Spanish Programa Nacional de Materiales under contract number MAT990569-C02. One of the authors (D.D.) wishes to acknowledge the Ministerio de Educacion y Cultura, Government of Spain for providing a fellowship. The authors are grateful to Dr. J. Camps for his comments about the chemistry of silicon.

${ }^{1}$ G. S. Selwyn, Jpn. J. Appl. Phys., Part 1 32, 3068 (1993).

${ }^{2}$ J. Dutta, I. M. Reaney, C. Bossel, R. Houriet, and H. Hofmann, Nanostruct. Mater. 6, 493 (1995).

${ }^{3}$ J. Costa, G. Sardin, J. Campmany, and E. Bertran, Vacuum 45, 1115 (1994).

${ }^{4}$ H. Hofmeister, J. Dutta, and H. Hofmann, Phys. Rev. B 54, 2856 (1996).

${ }^{5}$ J. Costa, P. Roura, J. R. Morante, and E. Bertran, J. Appl. Phys. 83, 7879 (1998).

${ }^{6}$ P. Roca i Cabarrocas, Mater. Res. Soc. Symp. Proc. 507, 855 (1998).

${ }^{7}$ G. Viera, P. Roca i Cabarrocas, S. Hamma, S. N. Sharma, J. Costa, and E. Bertran, Mater. Res. Soc. Symp. Proc. 467, 313 (1997).

${ }^{8}$ D. K. Nielsen, R. A. Street, C. C. Tsai, and J. C. Kights, Phys. Rev. B 20, 4839 (1979)

${ }^{9}$ J. Farjas, D. Das, J. Fort, and P. Roura, Phys. Rev. B (submitted).

${ }^{10}$ D. Das, J. Farjas, J. Costa, P. Roura, G. Viera, and E. Bertran, Mater. Res. Soc. Symp. Proc. 609, A5.11.1 (2000).

${ }^{11}$ E. Bertran, J. Costa, G. Sardin, J. Campmany, J. L. Andujar, and A. Canillas, Plasma Sources Sci. Technol. 3, 348 (1994).

${ }^{12}$ B. E. Deal and A. S. Grove, J. Appl. Phys. 36, 3770 (1965).

${ }^{13}$ J. Costa, J. Fort, J. J. Suñol, P. Roura, G. Viera, and E. Bertran, Mater. Res. Soc. Symp. Proc. 513, 427 (1998).

${ }^{14}$ D. Das, J. Farjas, P. Roura, G. Viera, and E. Bertran, Diamond Relat. Mater. 10, 1295 (2001)

${ }^{15}$ W. Beyer, J. Non-Cryst. Solids 266, 845 (2000).

${ }^{16}$ Y. L. Khait, R. Weil, R. Beserman, W. Beyer, and H. Wagner, Phys. Rev. B 42, 9000 (1990).

${ }^{17}$ M. L. Naiman, C. T. Kirk, B. L. Emerson, and J. B. Taitel, J. Appl. Phys. 58, 779 (1985).

${ }^{18}$ D. V. Tsu, G. Lukovski, and B. N. Davison, Phys. Rev. B 40, 1795 (1989). 